\title{
Metoprolol blunts the time-dependent progression of infarct size
}

\author{
Manuel Lobo-Gonzalez ${ }^{1,2,3} \cdot$ Carlos Galán-Arriola1,3 $\cdot$ Xavier Rossello ${ }^{1,3,4} \cdot$ Maribel González-Del-Hoyo ${ }^{1,5}$. \\ Jean Paul Vilchez ${ }^{1,6}$. María I. Higuero-Verdejo ${ }^{1,3}$. Jose M. García-Ruiz ${ }^{1,3,7}$. Gonzalo J. López-Martín ${ }^{1}$. \\ Javier Sánchez-González ${ }^{1,8} \cdot$ Eduardo Oliver $^{1,3} \cdot$ Gonzalo Pizarro ${ }^{1,3,6} \cdot$ Valentin Fuster $^{1,9} \cdot$ Borja Ibanez $^{1,2,3}$ (D)
}

Received: 18 May 2020 / Accepted: 20 July 2020 / Published online: 3 August 2020

(c) The Author(s) 2020

\begin{abstract}
Early metoprolol administration protects against myocardial ischemia-reperfusion injury, but its effect on infarct size progression (ischemic injury) is unknown. Eight groups of pigs (total $n=122$ ) underwent coronary artery occlusion of varying duration $(20,25,30,35,40,45,50$, or $60 \mathrm{~min})$ followed by reperfusion. In each group, pigs were randomized to i.v. metoprolol $(0.75 \mathrm{mg} / \mathrm{kg}$ ) or vehicle (saline) $20 \mathrm{~min}$ after ischemia onset. The primary outcome measure was infarct size (IS) on day7 cardiac magnetic resonance (CMR) normalized to area at risk (AAR, measured by perfusion computed tomography [CT] during ischemia). Metoprolol treatment reduced overall mortality ( $10 \%$ vs $26 \%, p=0.03)$ and the incidence and number of primary ventricular fibrillations during infarct induction. In controls, IS after 20 -min ischemia was $\approx 5 \%$ of the area AAR. Thereafter, IS progressed exponentially, occupying almost all the AAR after $35 \mathrm{~min}$ of ischemia. Metoprolol injection significantly reduced the slope of IS progression ( $p=0.004$ for final IS). Head-to-head comparison (metoprolol treated vs vehicle treated) showed statistically significant reductions in IS at 30,35, 40, and 50-min reperfusion. At 60-min reperfusion, IS was $100 \%$ of AAR in both groups. Despite more prolonged ischemia, metoprolol-treated pigs reperfused at $50 \mathrm{~min}$ had smaller infarcts than control pigs undergoing ischemia for 40 or $45 \mathrm{~min}$ and similar-sized infarcts to those undergoing 35 -min ischemia. Day-45 LVEF was higher in metoprolol-treated vs vehicle-treated pigs ( $41.6 \%$ vs $36.5 \%, p=0.008)$. In summary, metoprolol administration early during ischemia attenuates IS progression and reduces the incidence of primary ventricular fibrillation. These data identify metoprolol as an intervention ideally suited to the treatment of STEMI patients identified early in the course of infarction and requiring long transport times before primary angioplasty.
\end{abstract}

Keywords Acute myocardial infarction $\cdot$ Ischemia-reperfusion injury $\cdot$ Metoprolol $\cdot$ Early reperfusion

\section{Introduction}

A comment to this article is available at https://doi.org/10.1007/ s00395-020-0814-2

Borja Ibanez

bibanez@cnic.es

1 Translational Laboratory for Cardiovascular Imaging and Therapy, Centro Nacional de Investigaciones Cardiovasculares (CNIC), Madrid, Spain

2 Cardiology Department, IIS-Fundación Jiménez Díaz University Hospital, Madrid, Spain

3 Centro de Investigación Biomédica en Red en Enfermedades Cardiovasculares (CIBERCV), Madrid, Spain

4 Cardiology Department, IdISBa-Hospital Universitari Son Espases, Palma de Mallorca, Spain
Despite major improvements in reperfusion strategies and coadjuvant therapies, myocardial infarction (MI) remains a leading cause of mortality and morbidity worldwide [21,

5 Cardiology Department, University Hospital of Tarragona Joan XXIII, IISPV, Rovira i Virgili University, Tarragona, Spain

6 Complejo Hospitalario Ruber Juan Bravo, Madrid, Spain

7 Hospital de Cabueñes, Gijón, Spain

8 Philips Healthcare, Madrid, Spain

9 Icahn School of Medicine at Mount Sinai, New York, NY, USA 
30]. Infarct size (IS) is a major determinant of prognosis in MI survivors; therefore, interventions able to limit it are needed. Since the extent of irreversible injury progresses in a time-dependent manner, early blood flow restoration (reperfusion) is associated with lower IS, and this translates into better long-term outcomes $[3,33]$. This notion is the basis of the widely applied "time is muscle" principle [18]. If provided early, the best reperfusion strategy for patients presenting with a ST-elevation MI (STEMI) is primary percutaneous coronary intervention (PPCI). PPCI stops ischemic injury progression immediately and performs better than fibrinolysis in achieving adequate tissue perfusion and limiting microvascular injury. Unfortunately, PPCI is often not an immediate option, and reperfusion is delayed during patient transfer to a PCI facility. Current guidelines recommend PPCI if the anticipated time from STEMI diagnosis to wire crossing is $\leq 120 \mathrm{~min}$ [21]. If a longer transfer time is predicted, the recommended reperfusion strategy is systemic fibrinolysis. However, fibrinolysis results in complete reperfusion in only $\sim 50 \%$ of treated patients and exposes patients to potentially serious bleeding events, such as intracranial hemorrhage [21]. Interventions to delay the progression of ischemic damage could theoretically extend the 120 min window for selecting PPCI over fibrinolysis, allowing more patients to benefit from the best reperfusion strategy.

Intravenous (i.v.) administration of metoprolol during ongoing ischemia is associated with smaller infarcts, both in experimental models $[6,7,23]$ and in a recent clinical trial $[22,27]$. In experimental and clinical settings, the cardioprotective effect of i.v. metoprolol is dependent on the timing of administration; when injected very close to reperfusion, metoprolol has no infarct-limiting effect [7]. This timing-dependent cardioprotective effect might explain the neutral effects reported in another recent clinical trial [29]. The fact that i.v. metoprolol reduced IS only when injected long before reperfusion suggests that it might slow the rate of ischemic death (i.e. infarction progression).

To address this question, we studied the trajectories of IS progression in the presence or absence of i.v. metoprolol in a pig model of STEMI. To mimic the clinical scenario, i.v. metoprolol or vehicle was injected $20 \mathrm{~min}$ after coronary artery occlusion, and pigs were reperfused at different times, from immediately after injection to $40 \mathrm{~min}$ later (i.e. $60 \mathrm{~min}$ after ischemia onset). All pigs underwent cardiac magnetic resonance (CMR) imaging examinations at 7 and 45 days after STEMI induction.

\section{Methods}

The study was conducted at Centro Nacional de Investigaciones Cardiovasculares (CNIC) facilities and approved by Institutional and Regional Animal Research Committees. All animal procedures conformed to EU Directive 2010/63EU and Recommendation 2007/526/EC regarding the protection of animals used for experimental and other scientific purposes.

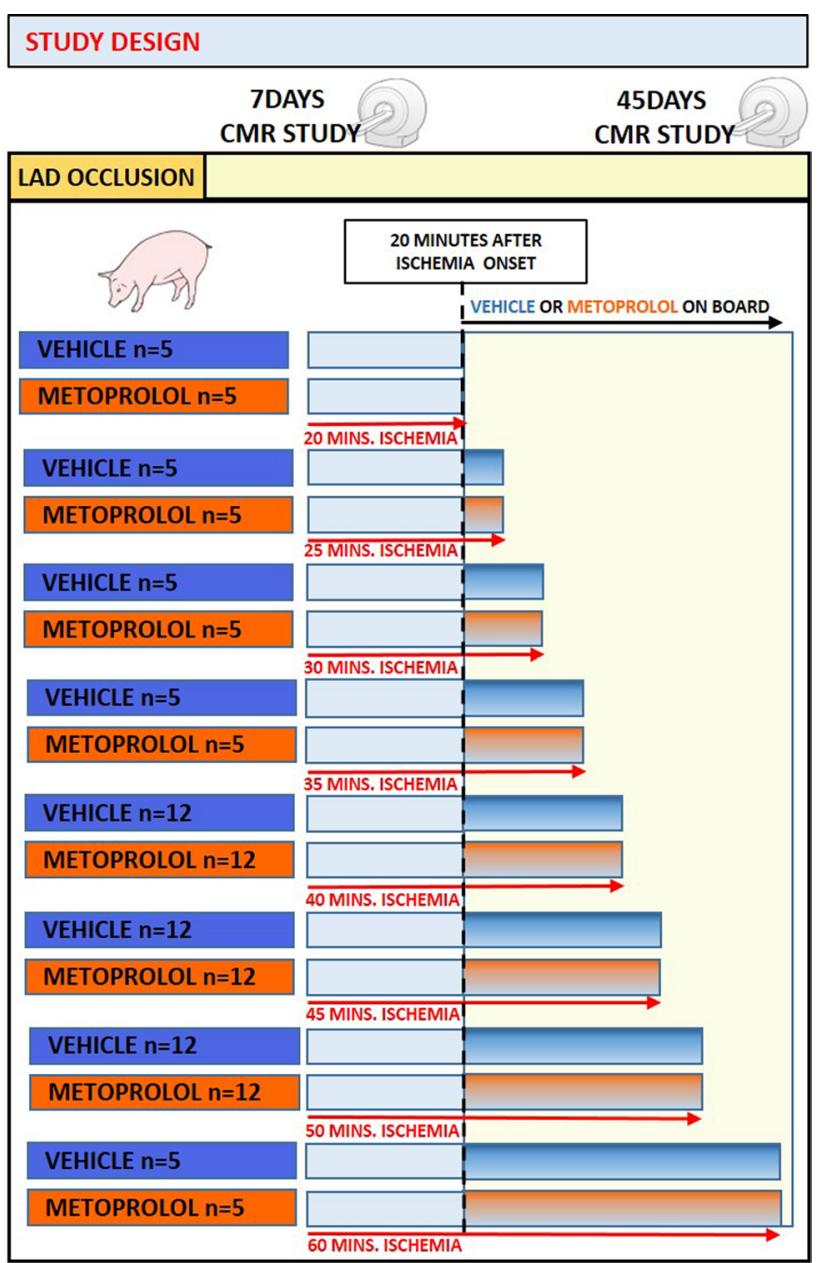

Fig. 1 Study design. Large white pigs $(30-35 \mathrm{~kg}$ ) were allocated to eight periods of ischemia by left anterior descending occlusion for $20(n=10), 25(n=10), 30(n=10), 35(n=10), 40 \quad(n=24), 45$ $(n=24), 50(n=24)$, or $60(n=10) \mathrm{min}$, followed by reperfusion. Animals were 1:1 randomized to receive either intravenous metoprolol $(0.75 \mathrm{mg} / \mathrm{kg})$ or saline $20 \mathrm{~min}$ after ischemia onset. MDCT scans were performed during the course of ischemia. Cardiac magnetic resonance scans were performed 7 days after reperfusion, and 45 days after reperfusion 


\section{Study design}

The study design is presented in Fig. 1. The study was performed in 3-month-old male large white pigs weighing $30-35 \mathrm{~kg}$ following state-of-the art methodologies [1, $17,25]$. Eight groups of pigs were scheduled to undergo ischemia for differing durations (20 min, $25 \mathrm{~min}, 30 \mathrm{~min}$, $35 \mathrm{~min}, 40 \mathrm{~min}, 45 \mathrm{~min}, 50 \mathrm{~min}$, or $60 \mathrm{~min}$ ). Ischemia was induced by inflating an angioplasty balloon in the mid-portion of the left anterior descending (LAD) coronary artery. At the end of the pre-specified ischemia duration, animals were reperfused (balloon deflation) and recovered from anesthesia. All pigs underwent contrast-enhanced multidetector computed tomography (MDCT) during coronary occlusion to depict the area at risk (AAR) [3]. All pigs were scheduled to undergo two CMR exams, at 7 and 45 days after infarction induction. Animals in each ischemia time group were randomly allocated to i.v. metoprolol $(0.75 \mathrm{mg} /$ $\mathrm{kg}$ of $1 \mathrm{mg} / \mathrm{ml}$ Beloken ${ }^{\circledR}$, Casen Recordati, Italy) [7] or i.v. vehicle $(0.75 \mathrm{ml} / \mathrm{kg}$ saline $)$. All metoprolol and vehicle injections were at 20 min after ischemia onset. Drug or vehicle was administered always at $20 \mathrm{~min}$ after the ischemia onset, in order to mimic the normal delay between symptoms and medical assistance in clinical arena. Operators were blinded to treatment allocation. The sample size of pigs scheduled to $40 \mathrm{~min}, 45 \mathrm{~min}$, and $50-\mathrm{min} \mathrm{I} / \mathrm{R}$ was $N=12$ per group, whereas sample size for the 20-min, 25-min, 30-min, 35 -min, and 60 -min I/R groups was $N=5$ per group. Dead animals were not replaced. All animals (including those allocated to vehicle during ischemia) received $50 \mathrm{mg}$ daily oral metoprolol throughout the study, starting the day after I/R induction. Clopidogrel $(150 \mathrm{mg})$ was administered immediately after reperfusion, followed by $75 \mathrm{mg}$ daily for two additional days.

The pre-specified primary endpoint IS [extent of late gadolinium enhancement (LGE) normalized to AAR] on day 7 CMR. Secondary outcomes were microvascular obstruction (MVO) on day 7 CMR as well as left ventricular (LV) volumes and LV systolic function (LV ejection fraction, LVEF) on day 45 . For comparative purposes, hemodynamic parameters (systolic and diastolic blood pressure) before ischemia onset and upon reperfusion, incidence and timing of ventricular fibrillation (VF) events, and mortality were recorded in all animals throughout the study.

\section{Pig model of STEMI (ischemia-reperfusion (I/R) protocol)}

The MI-induction protocol is detailed elsewhere [5, 32]. In brief, sedation was induced by intramuscular injection of ketamine $(20 \mathrm{mg} / \mathrm{kg})$, xylazine $(2 \mathrm{mg} / \mathrm{kg})$, and midazolam $(0.5 \mathrm{mg} / \mathrm{kg})$ and maintained by continuous intravenous infusion of ketamine $(2 \mathrm{mg} / \mathrm{kg} / \mathrm{h})$, xylazine $(0.2 \mathrm{mg} / \mathrm{kg} / \mathrm{h})$, and midazolam $(0.2 \mathrm{mg} / \mathrm{kg} / \mathrm{h})$. The analgesic buprenorphine $(0.03 \mathrm{mg} / \mathrm{kg})$ was administered by intramuscular injection immediately before the procedure. Animals were intubated and received mechanical ventilatory support with mandatory synchronized intermittent volume-control ventilation (fraction of inspired $\mathrm{O}_{2}, 28 \%$ ). Central venous and arterial lines were inserted, and a single bolus of unfractionated heparin $(300 \mathrm{IU} / \mathrm{kg})$ was administered at the onset of the instrumentation. During the procedure, animals were continuously infused with amiodarone $(300 \mathrm{mg} / \mathrm{h})$. Amiodarone was initiated immediately after coronary artery occlusion. Through the femoral arterial sheath, a guiding catheter was placed in the left main coronary artery. A coronary wire was placed distal in the LAD, and an angioplasty balloon was inflated in the mid-LAD, occluding the LAD immediately distal to the origin of the first diagonal branch. Balloon location, maintenance of inflation, and post-reperfusion patency were monitored by contrast angiography. In cases of VF, non-synchronized biphasic defibrillations were applied until sinus rhythm was restored.

\section{Arterial enhanced MDCT protocol and AAR quantification}

After coronary artery occlusion, pigs were moved to the MDCT suite. In all cases, pigs were back in the catheterization laboratory within $15 \mathrm{~min}$ of ischemia onset. Arterial phase MDCT studies were performed in a 64-slice CT scanner (Brilliance CT 64; Philips Healthcare, Cleveland, $\mathrm{OH})$ after intravenous administration of iodinated contrast medium. MDCT images were evaluated with dedicated software (MR Extended Work Space 2.6; Philips Healthcare, Best, The Netherlands) by two observers blinded to ischemia duration protocol and treatment allocation. Short-axis orientation images were obtained from volumetric CT images by multiplanar reconstruction. The region negative for contrast enhancement corresponds to the territory supplied by the occluded vessel (AAR) and was identified based on contrast enhancement differences vs the remote myocardium. The AAR was manually delineated and expressed as a percentage of LV area.

\section{CMR protocol and analysis}

Cine and contrast-enhanced CMR studies were performed 7 days and 45 days after MI induction. CMR examinations were conducted using a Philips 3-T Achieva Tx whole body scanner (Philips Healthcare, Best, The Netherlands) equipped with a 32-element phased-array cardiac coil. The imaging protocol included a standard segmented cine steady-state free-precession sequence to provide high-quality 
anatomic references and assessment of $\mathrm{LV}$ volumes, $\mathrm{LV}$ mass, and LVEF, as well as a T1-weighted inversion recovery turbo field echo sequence acquired 10-15 min after the administration of gadolinium contrast to assess IS (extent of LGE) and MVO (dark regions within the LGE region). CMR images were evaluated with dedicated software (MR Extended Work Space 2.6; QMassMR 7.6; Medis, Leiden, The Netherlands) by 2 observers experienced in CMR analysis and blinded to group allocation.

\section{Statistical analysis}

Sample size was calculated according to previous results, for a power $80 \%$, significance level of $5 \%$ and expected mortality of $30 \%$.

Categorical variables are expressed as percentages and were compared by the chi-square test (or the Fisher exact test when appropriate). Normal distribution of each data subset was checked using graphical methods and a Shapiro-Wilk test. Continuous variables are expressed as mean \pm standard deviation if normally distributed and otherwise as median (interquartile range [IQR]). Between-group comparisons were made by parametric methods (nonpaired Student's $t$ test) or nonparametric methods (Mann-Whitney $U$ test) as appropriate. Differences were considered statistically significant at $p<0.05$ (two tailed).

Cumulative mortality was calculated using the Kaplan-Meier method. The difference in survival estimates across dipstick categories was assessed by the log-rank test.

Slopes of myocardial damage over ischemia time in the vehicle and metoprolol groups were adjusted using an asymmetric sigmoidal model (least squares regression).

All statistical analyses were performed with Stata v15.1 (StataCorp, College Station, TX). Graphs were generated with GraphPad-Prism v7.0 (GraphPad Software, Inc, La Jolla, CA).

\section{Results}

Experimental MI was induced in 122 pigs. Of these, two animals died during MI induction due to refractory VF ( 1 in a metoprolol group 1 in a vehicle group). A further 4 animals died during the week after MI induction, before completing the 7-day CMR (1 in a metoprolol group, 3 in vehicle groups), and 14 animals died between day 7 and day 45, thus only undergoing the day 7 CMR (4 in metoprolol groups, 10 in vehicle groups). Therefore, overall mortality was $26.2 \%$ among vehicle-treated pigs vs. 9.8\% among metoprololtreated pigs $(p=0.07)$ (Fig. 2). There were no differences in hemodynamic parameters across treatment allocation for any ischemia duration (Table 1). Of note, the incidence and number of VF episodes during ongoing ischemia were

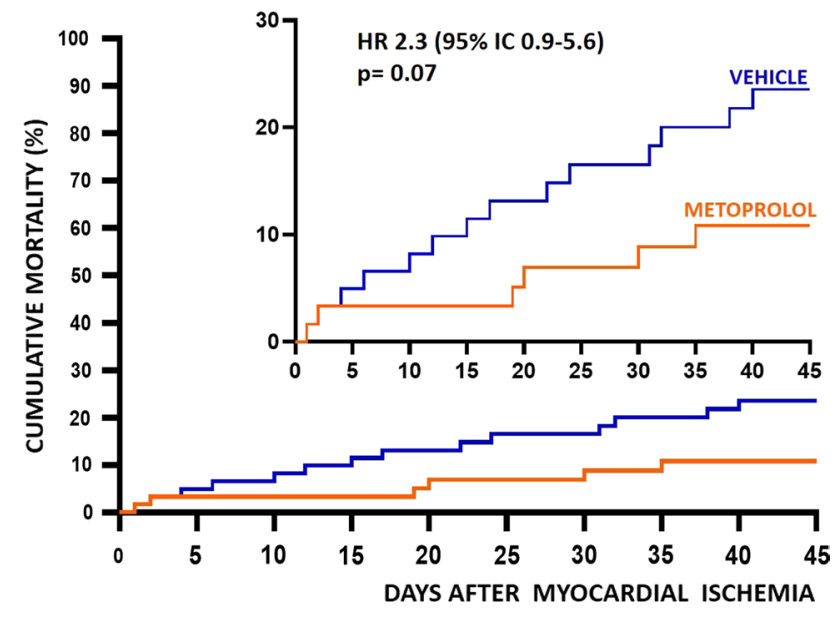

Fig. 2 Kaplan-Meier survival curves in pigs receiving metoprolol or vehicle during ischemia

Table 1 Hemodynamic parameters during myocardial infarction induction

\begin{tabular}{|c|c|c|c|}
\hline & Vehicle & Metoprolol & $p$ value \\
\hline \multicolumn{4}{|c|}{ Baseline (before myocardial infarction induction) } \\
\hline Heart rate (beats per min) & $76.6 \pm 9.5$ & $76.2 \pm 9.9$ & 0.860 \\
\hline Systolic blood pressure $(\mathrm{mmHg})$ & $113.1 \pm 8.0$ & $113.5 \pm 7.8$ & 0.821 \\
\hline $\begin{array}{l}\text { Diastolic blood pressure } \\
(\mathrm{mmHg})\end{array}$ & $71.5 \pm 5.8$ & $69.1 \pm 7.1$ & 0.079 \\
\hline \multicolumn{4}{|l|}{ Post-reperfusion } \\
\hline Heart rate (beats per min) & $91.8 \pm 9.6$ & $90.9 \pm 7.8$ & 0.612 \\
\hline Systolic blood pressure $(\mathrm{mmHg})$ & $88.8 \pm 9.6$ & $87.5 \pm 9.9$ & 0.498 \\
\hline $\begin{array}{l}\text { Diastolic blood pressure } \\
(\mathrm{mmHg})\end{array}$ & $58.8 \pm 6.3$ & $56.8 \pm 6.1$ & 0.125 \\
\hline
\end{tabular}

Data from animals in different ischemia duration protocols were pooled according to treatment allocation (metoprolol or vehicle)

Data are presented as mean \pm standard deviation unless otherwise noted

significantly lower in animals allocated to metoprolol (36.5\% for vehicle vs. $20.8 \%$ for metoprolol; $p=0.001$ ) (Fig. 3).

\section{Infarct size trajectories over time in the pig infarction-reperfusion model}

Infarct size was evaluated on day 7 CMR. Among pigs allocated to vehicle, infarctions in the 20 -min I/R protocol were very small (6.2 [0.0-9.7] \% AAR). Increases in ischemia duration from 20 to $35 \mathrm{~min}$ resulted is an exponential increase in IS (72.7 [69.0-87.3] \%AAR). An ischemia duration of 40 min resulted in a further increase in IS to occupy almost the entire AAR (97.8 [87.9-100.0] \% AAR) (Fig. 4). Absolute infarct size $(\% \mathrm{LV})$ followed the same temporal progression pattern (Fig. 5). 
Fig. 3 Incidence and timing of primary ventricular fibrillation. Left panel: percentage of animals with ventricular fibrillation (VF) per group (pooled data). The color scale ranking highlights the difference in $\mathrm{VF}$ incidence between pigs receiving saline and pigs receiving metoprolol. Right panel: plot of cumulative VF incidence (percentage of animal suffering at least one VF) against time from ischemia onset to first VF
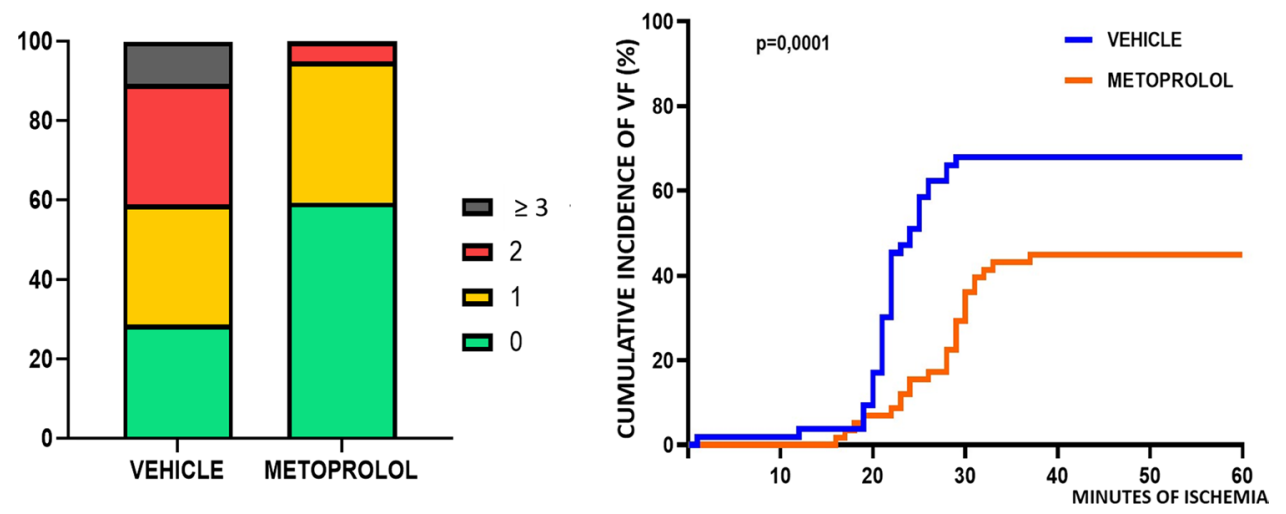

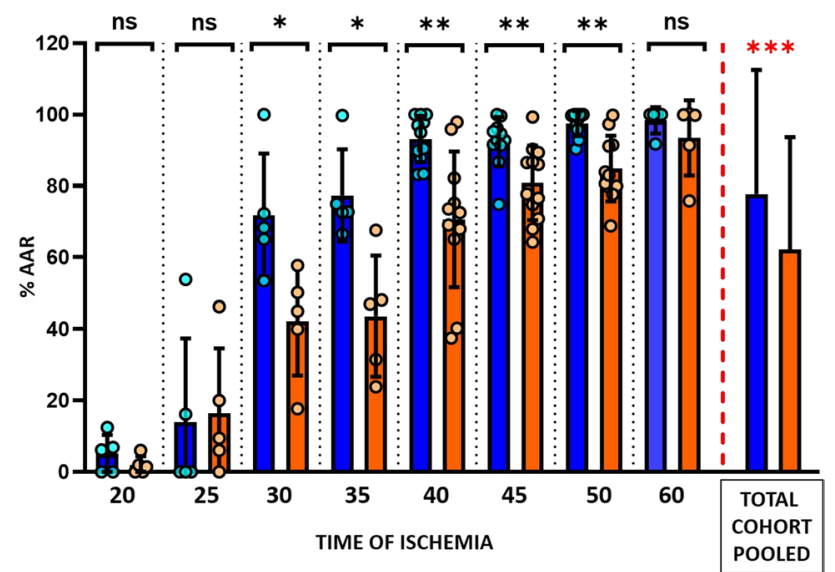

Fig. 4 Infarct size (normalized to area at risk) according to the duration of ischemia in metoprolol and vehicle-treated pigs. Infarct size as $\%$ of the AAR (area at risk) at 7-day CMR follow-up in groups allocated to different lengths of ischemia. Blue columns represent vehicle groups; orange columns represent metoprolol groups. Pooled total cohorts represent animals receiving vehicle (blue) versus animals receiving metoprolol (orange) irrespective of ischemia duration. Data are presented as median and IQR. Dots represent data for individual animals. $n s$ non-significant; $* p<0.05, * * p<0.01, * * * p<0.001$

\section{Impact of early i.v. metoprolol administration on infarct size trajectories}

For all ischemia durations, i.v. metoprolol was injected 20 min after ischemia onset in animals allocated to active treatment. In the 20 and 25 -min I/R groups, IS after metoprolol injection at min 20 did not differ significantly from that in vehicle-treated pigs (Figs. 4, 5). Infarcts in animals injected with metoprolol at min 20 and reperfusion at 30, 35, 40 , or $50 \mathrm{~min}$ after ischemia onset were consistently smaller than in vehicle-treated counterparts (Figs. 4, 5). The protection afforded by metoprolol was lost when reperfusion was delayed to $60 \mathrm{~min}$; pigs receiving metoprolol at $\min 20$ and reperfused at $60 \mathrm{~min}$ had virtually the same infarct size as their vehicle-treated counterparts. IS trajectories in vehicleand metoprolol-treated pigs are shown in Fig. 6.

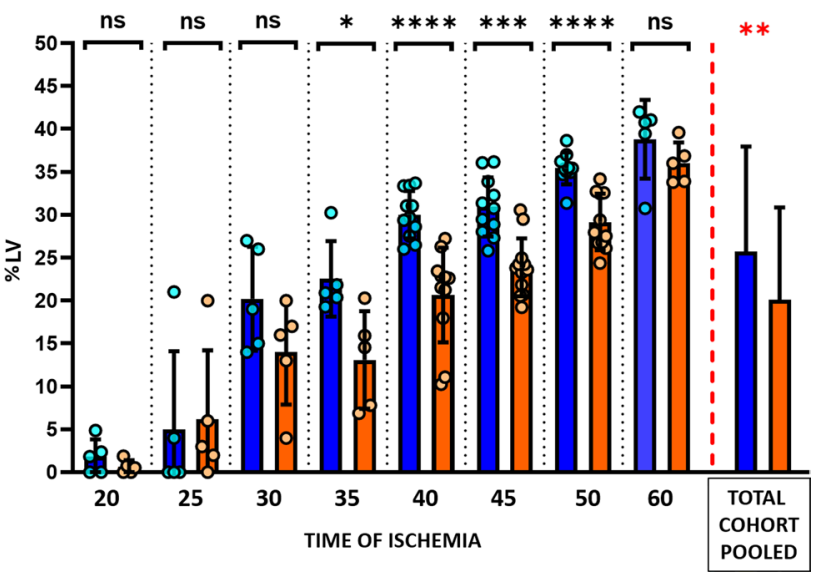

Fig. 5 Absolute infarct size according to duration of ischemia in metoprolol and vehicle-treated pigs. Infarct size as \% of the left ventricle at 7-day CMR follow-up in groups allocated to different lengths of ischemia. Blue columns represent vehicle groups; orange columns represent metoprolol groups. Pooled total cohorts represent animals receiving vehicle (blue) versus animals receiving metoprolol (orange) irrespective of ischemia duration. Data are presented as median and IQR. Dots represent data for individual animals. $n s$ non-significant; $* p<0.05, * * p<0.01, * * * p<0.001, * * * * p<0.0001$. $L V$ left ventricle

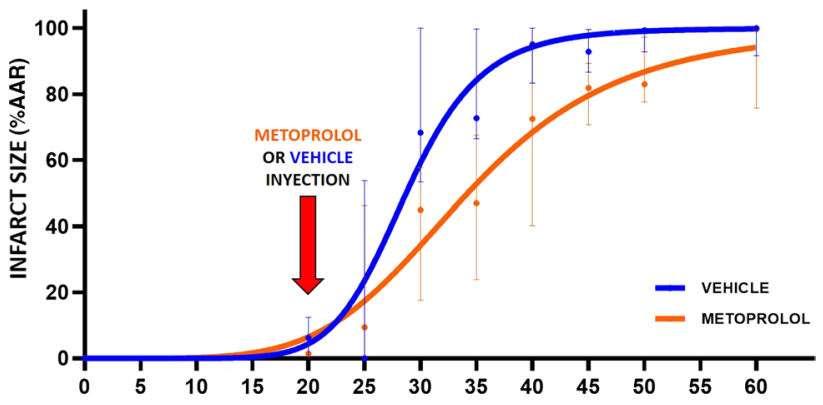

Fig. 6 Time-dependent progression of infarct size in the presence or absence of metoprolol. Slope progression of infarct size (\% AAR) with time of ischemia (minutes). Blue, vehicle group; orange, metoprolol group. Data are presented means (dots) \pm SD (lines) 


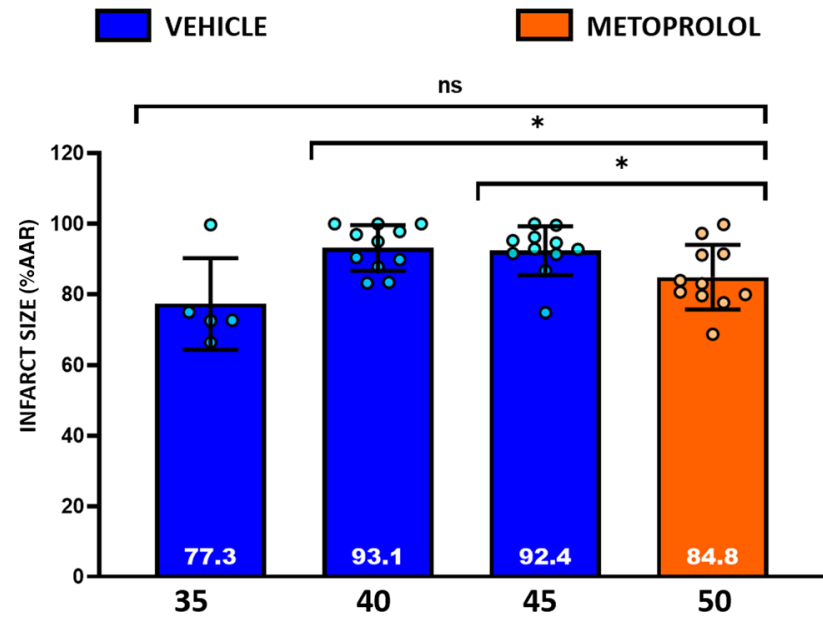

Fig. 7 Early administration of metoprolol delays infarct progression. Differences in IS (\% AAR) between animals receiving i.v. metoprolol and $50 \mathrm{~min}$ of ischemia and animals receiving i.v. vehicle and $35-$, $40-$, or 45 -min ischemia. $n s$ non-significant; $* p<0.05$, $* * p<0.01$, $* * * p<0.001, * * * * p<0.0001$

Pooling of data from all ischemia duration protocols revealed that pigs receiving i.v. metoprolol had significantly smaller relative infarctions (\% AAR) (Fig. 4) and absolute infarctions (\% LV) than those injected with vehicle (Fig. 5).

\section{Attenuation of infarct size progression by early i.v. metoprolol administration}

To evaluate the ischemic injury attenuation induced by early i.v. metoprolol injection in the course of an infarction, we compared IS in pigs undergoing 50-min I/R in the presence of metoprolol vs. those undergoing shorter I/R protocols without metoprolol. Infarct size (\%AAR) in pigs undergoing 50-min I/R and injected i.v. with metoprolol at ischemia min 20 had significantly smaller infarctions than vehicle-treated pigs undergoing 40 and $45-\mathrm{min} \mathrm{I} / \mathrm{R}$ protocols. IS (\%AAR) in metoprolol-treated pigs undergoing 50-min I/R did not differ from that in control pigs undergoing 35-min I/R (Fig. 7).

\section{Impact of early i.v. metoprolol administration on left ventricular ejection fraction and microvascular obstruction}

Long-term (day 45) LVEF was higher in metoprolol-treated pigs in all ischemia duration protocols, although statistical significance was reached only in the 35- and 40-min I/R groups. Pooling of data from all ischemia duration protocols revealed that pigs receiving i.v. metoprolol had significantly higher long-term LVEF than those injected with vehicle (Fig. 8). MVO g) on day 7 CMR did not differ between vehicle- and metoprolol-treated pigs. All CMR parameters

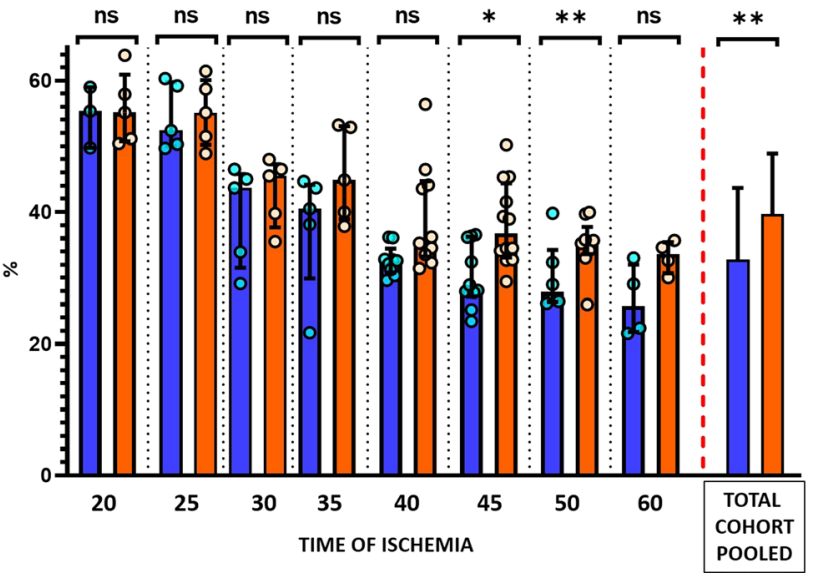

Fig. 8 Long-term left ventricular ejection fraction according to duration of ischemia in metoprolol and vehicle-treated pigs. LVEF at 45-day CMR follow-up in groups allocated to different lengths of ischemia. Blue, vehicle groups; orange, metoprolol groups. Pooled total cohorts represent animals receiving vehicle (blue) versus animals receiving metoprolol (orange) irrespective of ischemia duration. Data are presented as Median and IQR. Dots represent data for individual animals. $n s$ non-significant; ${ }^{*} p<0.05,{ }^{* *} p<0.01$

on day 7 and day 45 are shown for all ischemia duration protocols in Table 2.

\section{Discussion}

In the present study, we used a translational pig I/R model to determine the trajectories of infarct size progression in control conditions and in the presence of i.v. metoprolol delivered 20 min after ischemia onset. Reperfusion was initiated at different timings after ischemia onset, ranging from $20 \mathrm{~min}$ (immediately after vehicle/metoprolol injection) to $60 \mathrm{~min}$ (40 min after vehicle/metoprolol injection). CMR was performed on day 7 to quantify the extent of irreversible injury and on day 45 to measure long-term LVEF. The principal finding of the present study is that i.v. metoprolol significantly delays infarction progression (Fig. 6). These data identify i.v. metoprolol as one of the few interventions that can be initiated after ischemia onset able to reduce the progression of irreversible injury. To date, only reperfusion itself [28], and remote ischemic per-conditioning [24], were demonstrated to have this anti-ischemic damaging effect.

In STEMI survivors, the extent of irreversible injury (myocardial IS) is a strong predictor of long-term adverse events [20, 35]. Consequently, the extent of CMR-LGE (a surrogate for IS) is recommended as the primary outcome in clinical trials and large animal studies addressing the effect of cardioprotective strategies during STEMI [19]. Due to their independent association with outcomes, LVEF and MVO are also recommended as secondary outcomes [19]. 


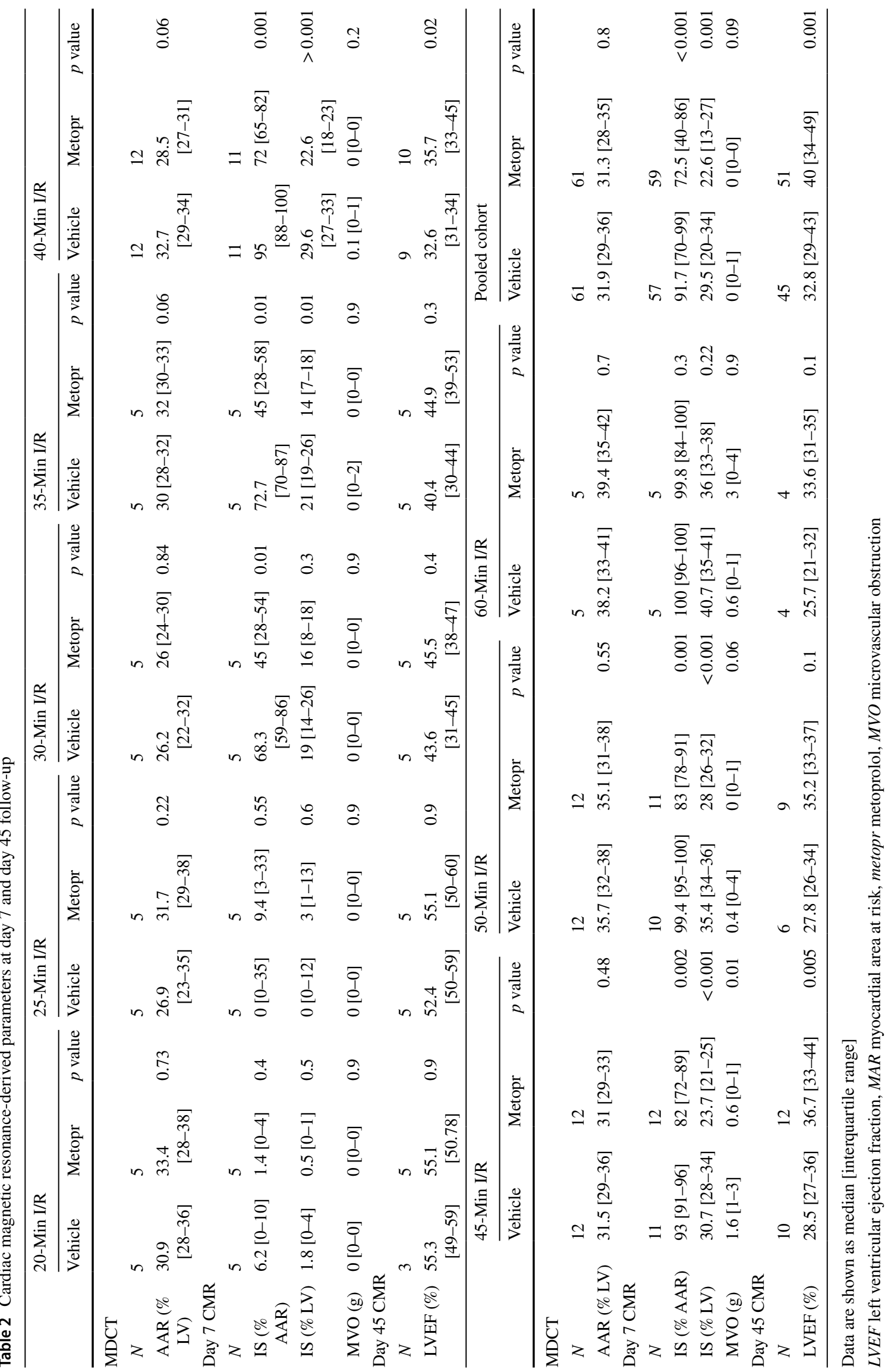


In the present study, we measured these three outcomes to evaluate the impact of metoprolol on irreversible myocardial damage. The beneficial effect of i.v. metoprolol injected during ischemia on all three surrogate markers strongly supports the potential of this intervention. It should be noted that despite metoprolol reduced MVO in some ischemia duration protocols, the incidence of this event was low in all preparations. It is well known that MVO is very frequent in different I/R models, including patients [12].

Classical studies using a large animal model of MI demonstrated that IS increases with time [28]. Other variables influencing the rate of irreversible injury progression include temperature and the presence of collaterals. Time-dependent IS progression was originally demonstrated in the dog [28], whose well-developed net of collaterals slows IS progression. Due to its anatomical and physiological similarities to humans, the large white pig is the most widely used I/R model [19]. Pigs lack collaterals, and IS progression is thus faster than in dogs. Our data show that most of the area subtended to ischemia becomes irreversibly injured between 20 and $35 \mathrm{~min}$ after ischemia onset. These data have important implications for future studies using this model because they show that, in the absence of strategies to alter ischemic injury, there is a very narrow time-window for reducing IS. Fast IS progression in humans usually occurs in young patients with no pre-existing collaterals, in whom large infarctions can be observed despite moderate ischemia durations [2]. This population is at much higher risk than older patients, who better tolerate prolonged ischemia. Timedependent IS progression is generally slower in the clinical setting than in the pig model used here, with IS progression taking at least $2 \mathrm{~h}$ to occupy most of the AAR [8]. We, therefore, hypothesize that the timing of metoprolol injection in our study (20 min after ischemia onset, or half the time taken for IS to represent most of the AAR in the pig) might be comparable to the clinical setting of a patient presenting during the first hour of STEMI (half the time taken for IS to represent most of the AAR in humans).

Interventions able to reduce IS beyond the effect of reperfusion itself would have major prognostic implications, and as such their identification is an unmet clinical need [21]. In the past, most tested interventions have targeted reperfusion injury, under the premise that this form of damage significantly contributes to final IS [13, 15]. Unfortunately, most clinical trials of these interventions have failed to demonstrate a clinical benefit [10, 16, 36]. There is, therefore, renewed interest in identifying strategies able to delay the IS progression (ischemic injury) [31]. In the present study, we show that metoprolol significantly attenuates the timedependent progression of IS (Fig. 6), thus positioning this strategy as one of few able to improve long-term outcomes in STEMI patients when applied in conjunction with reperfusion. Another intervention that has been shown to reduce ischemic injury is remote ischemic pre-conditioning. Kleinbongard et al. recently demonstrated in a similar pig model of reperfused MI that remote ischemic conditioning initiated after coronary occlusion resulted in attenuation of STsegment elevation despite ongoing coronary occlusion and this translated into smaller MI size [24]. These data strongly suggest that this intervention attenuated ischemic injury. In the clinical setting, remote ischemic per-conditioning has recently failed to improve clinical outcomes in STEMI patients [11]. However, it should be noted that patients enrolled in this trial in many centers were enrolled in the cath lab, and the time from conditioning onset to reperfusion was very short many times, leaving a very small room for ischemia progression attenuation.

The infarct-limiting effects of metoprolol in STEMI patients undergoing PPCI have been tested in two randomized clinical trials (the METOCARD-CNIC [22, 27] and the EARLY-BAMI trials [29]), which produced different outcomes [26]. In the METOCARD-CNIC trial, metoprolol administration was associated with smaller infarcts [22] and higher long-term LVEF [27], whereas in the EARLYBAMI study, metoprolol did not improve clinical outcomes [29]. In both trials, metoprolol was shown to be safe and to reduce the incidence of primary VF. An important difference between the trials is the timing of metoprolol administration. In METOCARD-CNIC, metoprolol was injected immediately after STEMI diagnosis, whereas in the EARLY-BAMI trial, some patients did not receive the full metoprolol dose, or received it when they reached the catheterization lab [26]. Our results reconcile the apparent contradictory trial findings and confirm that metoprolol most likely exerts its cardioprotective effect by slowing IS progression. Metoprolol is a potent protector against reperfusion injury in mouse models of I/R [6]; however, reperfusion injury appears to contribute less to final IS in pigs and humans than in rodents, and this difference might partially explain the failures to translate promising cardioprotective strategies to the clinic. In this regard, it has been proposed that there is a narrow ischemia duration window at which reperfusion-related injury significantly contributes to IS in large animals and humans [14]. If this was the case, part of the benefits of metoprolol seen in our study could have also been the result of reperfusioninjury amelioration, which was seen at ischemia duration protocols of 30-50 min, neither shorter nor longer than this window. In this regard, we previously demonstrated that part of the metoprolol-related infarct-limiting effects was driven by a direct effect on neutrophils and neutrophil-platelet interactions, reducing reperfusion-related injury [6]. If this was also a prevailing mechanism in the large animal model, it might be very well the case that to induce neutrophil stunning in a significant number of cells, metoprolol needs a time of circulation. This could partially explain the benefits 
observed only when metoprolol was on board $10 \mathrm{~min}$ or more.

One aspect than deserves explanation is the lack of differences between metoprolol and vehicle in terms of heart rate or blood pressure at reperfusion. Metoprolol has a known hemodynamic effect not observed in our experimental setting. The reason for this is the use of continuous amiodarone infusion $(300 \mathrm{mg} / \mathrm{h})$ in both treatment groups. Amiodarone has strong effects on heart rate and blood pressure. It is noteworthy that even in the absence of differences in heart rate, metoprolol administration was associated with significant IS-reducing effect. These data clearly dissociate the heart rate reducing effect to the cardioprotective one and explain why other drugs that lower heart rate, such as calcium channel blockers, are not associated with IS-reducing effect.

Reperfusion (either mechanical by PPCI or pharmacological by fibrinolysis) has become the mainstay treatment for STEMI patients. Head-to-head comparison of these approaches clearly favors PPCI, which results in a more complete reperfusion with less MVO and is associated with fewer complications. However, PPCI is not always an immediate option, and triaging patients for PPCI delays reperfusion. Current guidelines recommend transfer of patients to the PPCI center over immediate fibrinolysis so long as this can be done within 120 min of STEMI diagnosis [21]. The significant slowing of IS progression afforded by metoprolol could be especially beneficial for patients facing a long journey time to the PPCI center. Early administration of metoprolol, especially to patients presenting anterior infarctions, early in the course of STEMI (within 1-2 h from symptom onset), might extend the time window for selecting PPCI over fibrinolysis, thus allowing the best reperfusion strategy to be offered to patients who otherwise would receive fibrinolysis. As a limitation to this hypothesis the effects of metoprolol added to fibrinolytic therapy is unknown.

An additional benefit of metoprolol is the reduction in VF, shown here and in previous clinical trials [26]. In this regard, it has been shown in the pig model of reperfused MI that the incidence of VF (not the number of events or defibrillations) is associated with larger IS [34]. Skyschally et al. showed that VF is associated with larger AAR and lower residual myocardial blood flow [34]. Given that in our study AAR was not different between groups, it is highly plausible that metoprolol had an impact on residual blood myocardial flow and this resulted in a reduced incidence of VF, as well as in a reduction of the ischemic injury progression. Clinical practice guidelines indicate i.v. beta-blockers upon STEMI diagnosis with a class of recommendation IIa and level of evidence A [21]. However, this recommendation is rarely implemented [9]. This seems a lost opportunity for STEMI patients given the strong attenuation of time-dependent IS progression shown here for metoprolol, plus its safety when administered to stable patients (Killip class I-II) and its potency in reducing primary VF $[22,29]$. What is needed is a definitive randomized clinical trial enrolling patients presenting far from the PPCI center (e.g. at the limit of the 120 min delay) to demonstrate the clinical benefits of early i.v. metoprolol administration and thus convince the clinical community of the value of this therapeutic strategy.

\section{Limitations}

Our conclusions are limited by the use of the pig model. Pigs are widely used as a translational model because of the similarities of their hemodynamic parameters and heart and coronary anatomy to humans $[4,19]$. However, the pig heart has poor collateral flow, and this lack of collaterals may translate into a faster progression of myocardial damage than occurs in patients. Other potential limitation is referred to VF, interactions between metoprolol and amiodarone could affect this anti-arrhythmic effects.

\section{Conclusions}

Using a pig model of reperfused STEMI, we have shown that early i.v. injection of metoprolol is able to delay the time-dependent progression of irreversible myocardial damage. IS in pigs receiving metoprolol at 20-min ischemia and reperfused at $50 \mathrm{~min}$ was equal to that of vehicle-treated pigs undergoing ischemia for $35 \mathrm{~min}$ (30\% less ischemia time) and significantly smaller than vehicle-treated pigs reperfused at $40 \mathrm{~min}$. Early i.v. metoprolol injection in the course of experimental MI results in better long-term LVEF. Metoprolol significantly reduced the incidence of primary VF during ischemia and long-term mortality. Early i.v. metoprolol administration seems best suited for patients presenting early in the course of STEMI with a long anticipated transport time to the PPCI center.

Funding This study received funding from the Ministry of Science and Innovation ("RETOS 2019" Grant no. PID2019-107332RB-I00), from the Instituto de Salud Carlos III (ISCIII; PI16/02110) and the European Regional Development Fund (ERDF) "A way of making Europe" (\# AC16/00021), and from the Spanish Society of Cardiology through a 2017 Translational Research grant. BI has received funding from the European Research Council (ERC) under the European Union's Horizon 2020 research and innovation programme (ERC-Consolidator Grant agreement no. 819775). M.L received support from a 2015 Severo Ochoa CNIC intramural grant. X.R. received support from the SEC-CNIC CARDIOJOVEN fellowship program. R.F-J is a recipient of funding from the Carlos III Institute of Health-Fondo de Investigacion Sanitaria (PI19/01704) and has received funding from the European Union Horizon 2020 research and innovation programme under Marie Skłodowska-Curie grant agreement No 707642. EO is recipient of funds from Programa de Atracción de Talento (2017-T1/BMD-5185) of Comunidad de Madrid. The CNIC is supported by the ISCIII, the Ministerio de Ciencia e Innovación (MICINN) and the Pro CNIC Foundation, and is a Severo Ochoa Center of Excellence (SEV-2015-0505). 


\section{Compliance with ethical standards}

Conflict of interest Javier Sanchez-González is a Philips employee. All other authors state that they have no conflict of interests to declare.

Open Access This article is licensed under a Creative Commons Attribution 4.0 International License, which permits use, sharing, adaptation, distribution and reproduction in any medium or format, as long as you give appropriate credit to the original author(s) and the source, provide a link to the Creative Commons licence, and indicate if changes were made. The images or other third party material in this article are included in the article's Creative Commons licence, unless indicated otherwise in a credit line to the material. If material is not included in the article's Creative Commons licence and your intended use is not permitted by statutory regulation or exceeds the permitted use, you will need to obtain permission directly from the copyright holder. To view a copy of this licence, visit http://creativecommons.org/licenses/by/4.0/.

\section{References}

1. Botker HE, Hausenloy D, Andreadou I, Antonucci S, Boengler K, Davidson SM, Deshwal S, Devaux Y, Di Lisa F, Di Sante M, Efentakis P, Femmino S, Garcia-Dorado D, Giricz Z, Ibanez B, Iliodromitis E, Kaludercic N, Kleinbongard P, Neuhauser M, Ovize M, Pagliaro P, Rahbek-Schmidt M, Ruiz-Meana M, Schluter KD, Schulz R, Skyschally A, Wilder C, Yellon DM, Ferdinandy P, Heusch G (2018) Practical guidelines for rigor and reproducibility in preclinical and clinical studies on cardioprotection. Basic Res Cardiol 113:39. https://doi.org/10.1007/s00395-018-0696-8

2. Curran J, Burkhoff D, Kloner RA (2019) Beyond reperfusion: acute ventricular unloading and cardioprotection during myocardial infarction. J Cardiovasc Transl Res 12:95-106. https://doi. org/10.1007/s12265-019-9863-Z

3. Fernandez-Jimenez R, Galan-Arriola C, Sanchez-Gonzalez J, Aguero J, Lopez-Martin GJ, Gomez-Talavera S, Garcia-Prieto J, Benn A, Molina-Iracheta A, Barreiro-Perez M, Martin-Garcia A, Garcia-Lunar I, Pizarro G, Sanz J, Sanchez PL, Fuster V, Ibanez B (2017) Effect of ischemia duration and protective interventions on the temporal dynamics of tissue composition after myocardial infarction. Circ Res 121:439-450. https://doi.org/10.1161/CIRCR ESAHA.117.310901

4. Fernandez-Jimenez R, Ibanez B (2015) CAESAR: one step beyond in the construction of a translational bridge for cardioprotection. Circ Res 116:554-556. https://doi.org/10.1161/CIRCR ESAHA.115.305841

5. Fernandez-Jimenez R, Sanchez-Gonzalez J, Aaueero J, GarciaPrieto J, Lopez-Martin GJ, Garcia-Ruiz JM, Molina-Iracheta A, Rossello X, Fernandez-Friera L, Pizarro G, Garcia-Alvarez A, Dall'Armellina E, Macaya C, Choudhury RP, Fuster V, Ibanez B (2015) Myocardial edema after ischemia/reperfusion is not stable and follows a bimodal pattern imaging and histological tissue characterization. J Am Coll Cardiol 65:315-323. https:// doi.org/10.1016/j.jacc.2014.11.004

6. Garcia-Prieto J, Villena-Gutierrez R, Gomez M, Bernardo E, PunGarcia A, Garcia-Lunar I, Crainiciuc G, Fernandez-Jimenez R, Sreeramkumar V, Bourio-Martinez R, Garcia-Ruiz JM, Del Valle AS, Sanz-Rosa D, Pizarro G, Fernandez-Ortiz A, Hidalgo A, Fuster V, Ibanez B (2017) Neutrophil stunning by metoprolol reduces infarct size. Nat Commun 8:14780. https://doi.org/10.1038/ ncomms 14780

7. Garcia-Ruiz JM, Fernandez-Jimenez R, Garcia-Alvarez A, Pizarro G, Galan-Arriola C, Fernandez-Friera L, Mateos A, Nuno-Ayala M, Aguero J, Sanchez-Gonzalez J, Garcia-Prieto J, Lopez-Melgar
B, Martinez-Tenorio P, Lopez-Martin GJ, Macias A, PerezAsenjo B, Cabrera JA, Fernandez-Ortiz A, Fuster V, Ibanez B (2016) Impact of the timing of metoprolol administration during STEMI on infarct size and ventricular function. J Am Coll Cardiol 67:2093-2104. https://doi.org/10.1016/j.jacc.2016.02.050

8. Gersh BJ, Stone GW, White HD, Holmes DR Jr (2005) Pharmacological facilitation of primary percutaneous coronary intervention for acute myocardial infarction: is the slope of the curve the shape of the future? JAMA 293:979-986. https://doi.org/10.1001/ jama.293.8.979

9. Gibbons RJ, Miller TD (2017) Optimal medical therapy for known coronary artery disease: a review. JAMA Cardiol 2:1030-1035. https://doi.org/10.1001/jamacardio.2017.2249

10. Hausenloy DJ, Botker HE, Engstrom T, Erlinge D, Heusch G, Ibanez B, Kloner RA, Ovize M, Yellon DM, Garcia-Dorado D (2017) Targeting reperfusion injury in patients with ST-segment elevation myocardial infarction: trials and tribulations. Eur Heart J 38:935-941. https://doi.org/10.1093/eurheartj/ehw145

11. Hausenloy DJ, Kharbanda RK, Moller UK, Ramlall M, Aaroe J, Butler R, Bulluck H, Clayton T, Dana A, Dodd M, Engstrom T, Evans R, Lassen JF, Christensen EF, Garcia-Ruiz JM, Gorog DA, Hjort J, Houghton RF, Ibanez B, Knight R, Lippert FK, Lonborg JT, Maeng M, Milasinovic D, More R, Nicholas JM, Jensen LO, Perkins A, Radovanovic N, Rakhit RD, Ravkilde J, Ryding AD, Schmidt MR, Riddervold IS, Sorensen HT, Stankovic G, Varma M, Webb I, Terkelsen CJ, Greenwood JP, Yellon DM, Botker HE, Investigators C-E-P (2019) Effect of remote ischaemic conditioning on clinical outcomes in patients with acute myocardial infarction (CONDI-2/ERIC-PPCI): a single-blind randomised controlled trial. Lancet. https://doi.org/10.1016/S0140 -6736(19)32039-2

12. Heusch $\mathrm{G}$ (2016) The coronary circulation as a target of cardioprotection. Circ Res 118:1643-1658. https://doi.org/10.1161/CIRCR ESAHA.116.308640

13. Heusch G (2017) Critical issues for the translation of cardioprotection. Circ Res 120:1477-1486. https://doi.org/10.1161/CIRCR ESAHA.117.310820

14. Heusch G (2015) Treatment of myocardial ischemia/reperfusion injury by ischemic and pharmacological postconditioning. Compr Physiol 5:1123-1145. https://doi.org/10.1002/cphy.c140075

15. Heusch G, Gersh BJ (2017) The pathophysiology of acute myocardial infarction and strategies of protection beyond reperfusion: a continual challenge. Eur Heart J 38:774-784. https://doi. org/10.1093/eurheartj/ehw224

16. Heusch G, Rassaf T (2016) Time to give up on cardioprotection? A critical appraisal of clinical studies on ischemic pre-, post-, and remote conditioning. Circ Res 119:676-695. https://doi. org/10.1161/CIRCRESAHA.116.308736

17. Heusch G, Skyschally A, Schulz R (2011) The in-situ pig heart with regional ischemia/reperfusion-ready for translation. $\mathrm{J}$ Mol Cell Cardiol 50:951-963. https://doi.org/10.1016/j.yjmcc .2011 .02 .016

18. Horneffer PJ, Healy B, Gott VL, Gardner TJ (1987) The rapid evolution of a myocardial infarction in an end-artery coronary preparation. Circulation 76:V39-V42

19. Ibanez B, Aletras AH, Arai AE, Arheden H, Bax J, Berry C, Bucciarelli-Ducci C, Croisille P, Dall'Armellina E, Dharmakumar R, Eitel I, Fernandez-Jimenez R, Friedrich MG, Garcia-Dorado D, Hausenloy DJ, Kim RJ, Kozerke S, Kramer CM, Salerno M, Sanchez-Gonzalez J, Sanz J, Fuster V (2019) Cardiac MRI endpoints in myocardial infarction experimental and clinical trials: JACC scientific expert panel. J Am Coll Cardiol 74:238-256. https ://doi.org/10.1016/j.jacc.2019.05.024

20. Ibanez B, Heusch G, Ovize M, Van de Werf F (2015) Evolving therapies for myocardial ischemia/reperfusion injury. J Am Coll Cardiol 65:1454-1471. https://doi.org/10.1016/j.jacc.2015.02.032 
21. Ibanez B, James S, Agewall S, Antunes MJ, Bucciarelli-Ducci C, Bueno H, Caforio ALP, Crea F, Goudevenos JA, Halvorsen S, Hindricks G, Kastrati A, Lenzen MJ, Prescott E, Roffi M, Valgimigli M, Varenhorst C, Vranckx P, Widimsky P, Group ESCSD (2018) 2017 ESC Guidelines for the management of acute myocardial infarction in patients presenting with ST-segment elevation: The Task Force for the management of acute myocardial infarction in patients presenting with ST-segment elevation of the European Society of Cardiology (ESC). Eur Heart J 39:119-177. https://doi.org/10.1093/eurheartj/ehx393

22. Ibanez B, Macaya C, Sanchez-Brunete V, Pizarro G, FernandezFriera L, Mateos A, Fernandez-Ortiz A, Garcia-Ruiz JM, GarciaAlvarez A, Iniguez A, Jimenez-Borreguero J, Lopez-Romero P, Fernandez-Jimenez R, Goicolea J, Ruiz-Mateos B, Bastante T, Arias M, Iglesias-Vazquez JA, Rodriguez MD, Escalera N, Acebal C, Cabrera JA, Valenciano J, Perez de Prado A, Fernandez-Campos MJ, Casado I, Garcia-Rubira JC, Garcia-Prieto J, Sanz-Rosa D, Cuellas C, Hernandez-Antolin R, Albarran A, Fernandez-Vazquez F, de la Torre-Hernandez JM, Pocock S, Sanz G, Fuster V (2013) Effect of early metoprolol on infarct size in ST-segment-elevation myocardial infarction patients undergoing primary percutaneous coronary intervention: the effect of metoprolol in cardioprotection during an acute myocardial infarction (METOCARD-CNIC) Trial. Circulation 128:1495-1503. https:// doi.org/10.1161/CIRCULATIONAHA.113.003653

23. Ibanez B, Prat-Gonzalez S, Speidl WS, Vilahur G, Pinero A, Cimmino G, Garcia MJ, Fuster V, Sanz J, Badimon JJ (2007) Early metoprolol administration before coronary reperfusion results in increased myocardial salvage: analysis of ischemic myocardium at risk using cardiac magnetic resonance. Circulation 115:29092916. https://doi.org/10.1161/CIRCULATIONAHA.106.679639

24. Kleinbongard P, Amanakis G, Skyschally A, Heusch G (2018) Reflection of cardioprotection by remote ischemic perconditioning in attenuated ST-segment elevation during ongoing coronary occlusion in pigs: evidence for cardioprotection from ischemic injury. Circ Res 122:1102-1108. https://doi.org/10.1161/CIRCR ESAHA.118.312784

25. Lindsey ML, Bolli R, Canty JM Jr, Du XJ, Frangogiannis NG, Frantz S, Gourdie RG, Holmes JW, Jones SP, Kloner RA, Lefer DJ, Liao R, Murphy E, Ping P, Przyklenk K, Recchia FA, Schwartz Longacre L, Ripplinger CM, Van Eyk JE, Heusch G (2018) Guidelines for experimental models of myocardial ischemia and infarction. Am J Physiol Heart Circ Physiol 314:H812-H838. https://doi.org/10.1152/ajpheart.00335.2017

26. Martinez-Milla J, Raposeiras-Roubin S, Pascual-Figal DA, Ibanez B (2019) Role of beta-blockers in cardiovascular disease in 2019. Rev Esp Cardiol (Engl Ed) 72:844-852. https://doi.org/10.1016/j. rec.2019.04.014

27. Pizarro G, Fernandez-Friera L, Fuster V, Fernandez-Jimenez R, Garcia-Ruiz JM, Garcia-Alvarez A, Mateos A, Barreiro MV, Escalera N, Rodriguez MD, de Miguel A, Garcia-Lunar I, ParraFuertes JJ, Sanchez-Gonzalez J, Pardillos L, Nieto B, Jimenez A, Abejon R, Bastante T, Martinez de Vega V, Cabrera JA, LopezMelgar B, Guzman G, Garcia-Prieto J, Mirelis JG, Zamorano JL, Albarran A, Goicolea J, Escaned J, Pocock S, Iniguez A, Fernandez-Ortiz A, Sanchez-Brunete V, Macaya C, Ibanez B (2014) Long-term benefit of early pre-reperfusion metoprolol administration in patients with acute myocardial infarction: results from the METOCARD-CNIC trial (effect of metoprolol in cardioprotection during an acute myocardial infarction). J Am Coll Cardiol 63:2356-2362. https://doi.org/10.1016/j.jacc.2014.03.014

28. Reimer KA, Lowe JE, Rasmussen MM, Jennings RB (1977) The wavefront phenomenon of ischemic cell death. 1. Myocardial infarct size vs duration of coronary occlusion in dogs. Circulation 56:786-794

29. Roolvink V, Ibanez B, Ottervanger JP, Pizarro G, van Royen N, Mateos A, Dambrink JH, Escalera N, Lipsic E, Albarran A, Fernandez-Ortiz A, Fernandez-Aviles F, Goicolea J, Botas J, Remkes W, Hernandez-Jaras V, Kedhi E, Zamorano JL, Navarro F, Alfonso F, Garcia-Lledo A, Alonso J, van Leeuwen M, Nijveldt R, Postma S, Kolkman E, Gosselink M, de Smet B, Rasoul S, Piek JJ, Fuster V, van't Hof AW, Investigators E-B (2016) Early intravenous beta-blockers in patients with ST-segment elevation myocardial infarction before primary percutaneous coronary intervention. J Am Coll Cardiol 67:2705-2715. https://doi.org/10.1016/j. jacc.2016.03.522

30. Rossello X, Huo Y, Pocock S, Van de Werf F, Chin CT, Danchin N, Lee SW, Medina J, Vega A, Bueno H (2017) Global geographical variations in ST-segment elevation myocardial infarction management and post-discharge mortality. Int J Cardiol 245:27-34. https://doi.org/10.1016/j.ijcard.2017.07.039

31. Rossello X, Ibanez B (2018) Infarct size reduction by targeting ischemic injury: back to square one. Circ Res 122:1041-1043. https://doi.org/10.1161/CIRCRESAHA.118.312939

32. Rossello X, Pinero A, Fernandez-Jimenez R, Sanchez-Gonzalez J, Pizarro G, Galan-Arriola C, Lobo-Gonzalez M, Vilchez JP, Garcia-Prieto J, Garcia-Ruiz JM, Garcia-Alvarez A, Sanz-Rosa D, Ibanez B (2018) Mirabegron, a clinically approved beta3 adrenergic receptor agonist, does not reduce infarct size in a swine model of reperfused myocardial infarction. J Cardiovasc Transl Res 11:310-318. https://doi.org/10.1007/s12265-018-9819-8

33. Shiomi H, Nakagawa Y, Morimoto T, Furukawa Y, Nakano A, Shirai S, Taniguchi R, Yamaji K, Nagao K, Suyama T, Mitsuoka H, Araki M, Takashima H, Mizoguchi T, Eisawa H, Sugiyama S, Kimura T, Investigators CR-KA (2012) Association of onset to balloon and door to balloon time with long term clinical outcome in patients with ST elevation acute myocardial infarction having primary percutaneous coronary intervention: observational study. BMJ 344:e3257. https://doi.org/10.1136/bmj.e3257

34. Skyschally A, Amanakis G, Neuhauser M, Kleinbongard P, Heusch G (2017) Impact of electrical defibrillation on infarct size and no-reflow in pigs subjected to myocardial ischemia-reperfusion without and with ischemic conditioning. Am J Physiol Heart Circ Physiol 313:H871-H878. https://doi.org/10.1152/ajpheart.00293 .2017

35. Stone GW, Selker HP, Thiele H, Patel MR, Udelson JE, Ohman EM, Maehara A, Eitel I, Granger CB, Jenkins PL, Nichols M, BenYehuda O (2016) Relationship between infarct size and outcomes following primary PCI: patient-level analysis from 10 randomized trials. J Am Coll Cardiol 67:1674-1683. https://doi.org/10.1016/j. jacc.2016.01.069

36. Vanezis AP (2019) The broken promise of remote ischaemic conditioning. Lancet 394:1389-1390. https://doi.org/10.1016/S0140 $-6736(19) 32047-1$ 